\title{
Besser Standardhygiene-Netzwerke als MRSA-Netzwerke
}

\author{
Ines Kappstein
}

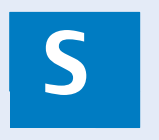

eit einiger Zeit werden vielerorts regionale MRSA-Netzwerke etabliert, um die Kommunikation und Zusammenarbeit zwischen Krankenhäusern, anderen Einrichtungen des Gesundheitssystems und weiterbehandelnden Ärzten zu fördern. Dabei sollen aufeinander abgestimmte Konzepte zu einer erfolgreichen Prävention von MRSA und nosokomialen MRSA-Infektionen führen. Allerdings ist bekannt, dass es eine Vielzahl anderer (multi-)resistenter Erreger (MRE) gibt, die heutzutage sogar mehr Behandlungsprobleme mit sich bringen als MRSA. Denn wir haben seit Anfang der 2000er-Jahre mittlerweile 4 neue Antibiotika mit Wirksamkeit gegen MRSA. Demgegenüber stehen gramnegative MRE, für die es derzeit kaum noch Therapieoptionen gibt.

Was also sollen MRSA-Netzwerke bewirken können? Soll MRSA vielleicht als pars pro toto fungieren, sollen also die Maßnahmen, die für die Prävention von MRSA seit über 10 Jahren von der Kommission für Krankenhaushygiene und Infektionsprävention (KRINKO) beim Robert-Koch-Institut (RKI) empfohlen werden, auch auf die anderen MRE übertragen werden? Was würde das für die Krankenversorgung in unseren Kliniken und die Rehabilitationsmaßnahmen in den nachsorgenden Einrichtungen bedeuten? Sollen dann also beispielsweise die für MRSA empfohlenen Screening-Maßnahmen auch auf die ESBL-Bildner mit Abstrichuntersuchungen an deren Prädilektionsstellen im Körper (vor allem Darm) ausgedehnt werden? Und sollen dann etwa alle Patienten mit Nachweis von MRE isoliert werden, wie für MRSA von der KRINKO empfohlen, bis der Erreger nicht mehr nachweisbar ist? Wie soll dann aber die Krankenversorgung in unseren Kliniken noch möglich sein, in denen es nur selten Einzelzimmer gibt? Wo soll das Personal herkommen, das die zeitaufwendigen mit der Isolierung zusammenhängenden Maßnahmen praktizieren soll? Und gibt es überhaupt wirksame Dekolonisierungsmaßnahmen? Viele Fragen, auf die es eigentlich kaum wirklich überzeugende Antworten gibt.
Und darüber hinaus: Was soll mit der Mehrzahl der Patienten geschehen, die - wie nun einmal alle Menschen - mit einer Vielzahl potenzieller Infektionserreger in ihrer Körperflora besiedelt sind? Auch diese i.d.R. nicht oder nur mäßig resistenten Mikroorganismen können übertragen werden und zwar auf den gleichen Wegen wie MRSA oder andere MRE - und können nosokomiale Infektionen (NI) verursachen. Wenn also die Übertragung von MRSA nur mit Maßnahmen vermeidbar sein sollen, die über die Standardhygienemaßnahmen hinausgehen (wie insbesondere Isolierung), dann müssen diese zusätzlichen Maßnahmen folgerichtig auf alle Patienten angewendet werden. Denn die weit überwiegende Mehrzahl der Patienten mit NI hat Infektionen (auch chronisch verlaufende) durch empfindliche oder nur geringfügig resistente Erreger - und leidet und stirbt ggf. auch an diesen Infektionen, obwohl es wirksame Antibiotika gibt. Man kann deshalb keineswegs sagen, dass diese Infektionen nicht so schwerwiegend oder gefährlich seien, nur weil es prinzipiell wirksame Antibiotika gibt. Die Befürworter der Isolierung argumentieren jedoch vor allem damit, dass man bei den resistenten Erregern deshalb spezielle Maßnahmen brauche, weil es im Falle einer Infektion zu wenige wirksame Antibiotika gebe und diese Infektionen deshalb gefährlicher seien. Wir wissen aber, um auf MRSA zurückzukommen, dass die Mehrzahl der auch lebensbedrohlichen Staphylococcus aureus-Infektionen durch normal empfindliche S. aureus (MSSA) verursacht werden, und wir wissen ferner, dass viele Menschen mit MSSA besiedelt sind. Müsste man dann nicht bei jedem Patienten ein Screening auf MRSA und MSSA durchführen? Wäre es somit nicht sogar unethisch, die Isolierung auf Patienten mit MRSA zu beschränken, wenn doch wesentlich mehr Patienten von NI durch MSSA betroffen sind?

Mit Hinweisen auf unethisches Verhalten oder daraus abgeleiteten Ansichten kommen wir aber ersichtlich nicht weiter bei solchen Fragen, die sich nur mit handfesten wissenschaftlichen Daten beantworten lassen. Das Vorhandensein solcher Daten ist eine Vorausset- 
zung für einen „Standard“ [1,2], der grundsätzlich einzuhalten und von dem abzuweichen nur unter bestimmten Umständen möglich ist. Ein solcher Standard wird aber nicht dadurch geschaffen, dass eine Expertenkommission eine Empfehlung abgibt, die nicht „evidence-based“ ist, wie das die KRINKO vor mehr als 10 Jahren mit ihrer Empfehlung zur MRSA-Prävention getan hat [3]. Diese kaum belegte Empfehlung mag der damaligen Situation mit nur einer verfügbaren wirksamen Antibiotikumgruppe geschuldet gewesen sein. Die wissenschaftliche Datenlage zur Isolierung bei MRSANachweis wird im aktuellen HTA-Bericht so zusammengefasst: „Die Auswertung der Studienergebnisse macht deutlich, dass die Frage, ob Isolierungsmaßnahmen an sich eine Effektivität bzgl. der Verringerung der MRSA-Raten besitzen, nicht abschließend beantwortet werden kann, da die verfügbare Literatur stark limitiert ist“ [4]. Ähnlich hat sich übrigens der Vorstand der Deutschen Gesellschaft für Krankenhaushygiene (DGKH) vor kurzem zur Isolierung geäußert: „Da bei der Prävention und Kontrolle immer ein ganzes Paket von Maßnahmen umgesetzt wird [...], ist bislang der individuelle Beitrag einzelner Komponenten im Multibarrierenkonzept gegen die nosokomiale MRSA-Ausbreitung nicht durch prospektiv randomisierte Studien belegt" [5].

Diese unklare wissenschaftliche Datenlage wirft auch die Frage auf, ob es vertretbar ist, Patienten mit MRSA wochen- und monatelang zu isolieren (S. aureus kann den Menschen sehr lange besiedeln) und so von ihrem privaten Umfeld in krasser Weise zu distanzieren, ihre Angehörigen und Freunde zu zwingen, sie nur noch in aufwendiger Schutzkleidung sehen zu dürfen, und sie in der Isolierung, also einsam, ihren Lebensabend verbringen und sterben zu lassen, obwohl man doch weiß, dass allein schon ein normaler Klinikaufenthalt insbesondere ältere Menschen enorm beeinträchtigt. Soll es vor diesem Hintergrund ferner vertretbar sein, Menschen derart an den Rand ihrer psychischen Stabilität zu bringen, dass sie den Suizid einem Weiterleben unter den Bedingungen der Isolierung vorziehen (auch das kommt vor)? Dazu hat der HTA-Bericht ebenfalls explizit Stellung genommen und ausgeführt: „Dringend erforderlich ist eine verbesserte Studienlage [...] adverser Nebeneffekte von [...] Kontaktschutzisolierungen [...]. Die psychosozialen Effekte einer MRSAInfektion sind in Deutschland völlig unzulänglich erforscht" [4].

Die in Deutschland (aber nicht überall in gleichem Maße und in den letzten Jahren stagnierend) in den letzten 2 Jahrzehnten gestiegenen MRSA-Raten werden von den Befürwortern der Isolierung häufig darauf zurückgeführt, dass die KRINKO-Empfehlungen nur unzureichend umgesetzt würden. Die meisten Kliniken geben aber bei Umfragen an, sich an die Empfehlungen der „RKI-Richtlinie“ zu halten [6]. Möchte man denn annehmen, dass dies nur bei Umfragen behauptet, aber nicht praktiziert wird? Wohl nicht. Müsste man also nicht auch zumindest als Möglichkeit in Betracht ziehen, dass diese Maßnahmen nicht wirksam sind? Das könne nicht sein, weil doch in den Niederlanden, wo seit Jahren die „Search-and-destroy“-Strategie praktiziert wird, MRSA viel seltener sei als bei uns? Neben „Search-and-destroy“ gibt es dort aber anders als bei uns eine breite Versorgung mit klinischen Infektiologen, Mikrobiologen bzw. Krankenhaushygienikern und mit dem restriktiven Einsatz von Antibiotika eine weitere Säule, die - international unbestritten - von immenser Bedeutung ist, um die Zunahme resistenter Erreger zu begrenzen [7].

Zum Schluss nun die Frage, ob MRSA-Netzwerke sinnvoll sind, wenn sie nur auf die Umsetzung der von der KRINKO 1999 empfohlenen Maßnahmen ausgerichtet sind. Ich meine: nein. Jedoch sind Netzwerke zur Prävention von NI nicht etwa generell nutzlos, sie sollten sich aber nicht auf MRE oder gar nur auf MRSA beschränken, sondern sich die Förderung der Infektionsprävention bei der medizinischen Versorgung generell zum Ziel setzen und insbesondere die Standardhygiene [8] bei der Versorgung aller Patienten in den Mittelpunkt stellen. Eine Isolierung von Patienten (z. B. bei Gastroenteritis) in Form einer vorübergehenden räumlichen Distanzierung von den anderen Patienten (durch Unterbringung in einem Einzelzimmer, das aber vom Personal und den Besuchern ohne Schutzkleidung betreten werden kann) mit den abhängig von der klinischen Situation jeweils sinnvollen patientennah praktizierten Schutzmaßnahmen ist, wenn überhaupt, vor allem nur kurzzeitig erforderlich [9]. Solche Netzwerke zu unterstützen, seine Fachkompetenz als Krankenhaushygieniker oder Hygienefachkraft einzubringen und die Erfahrungen der Beteiligten aus den anderen betroffenen Einrichtungen dabei einzubeziehen, wäre nicht nur generell für den Schutz von Patienten vor Infektionen sinnvoll, sondern auch für die eigene Arbeit bereichernd. Auf diese Weise würde sich ein umfassendes Verständnis für den Infektionsschutz bei der medizinischen Versorgung entwickeln können, denn das Konzept der Standardhygiene kann man jedem verständlich machen, ganz gleich ob Arzt, Pflegepersonal, Personal in Altenheimen oder Transportpersonal, weil es in sich schlüssig ist. 


\section{Literatur}

1 Ulsenheimer K. Haftungsrechtliche Bedeutung von Leitlinien. Krankenhaushyg up2date 2006; 1: 169 175

2 Klein M. Haftungsfragen im Hygienebereich - Hygienemängel gelten juristisch als „vollbeherrschbare Risiken“. Hyg \& Med 2010; 35: 361 - 366

3 Kappstein I. Empfehlungen der „Richtlinie“ - Was mache ich anders? Teil 3. Krankenhaushyg up2date 2009; 4: 125 - 137

4 Korczak D, Schöffmann C. Medizinische Wirksamkeit und Kosteneffektivität von Präventions- und Kontrollmaßnahmen gegen Methicillin-resistente Staphylococcus aureus (MRSA)-Infektionen im Krankenhaus. Schriftenreihe Health-Technology Assessment (HTA) in der BRD, DAHTA des DIMDI, Band 100, 2010 (www.dimdi.de)
5 Simon A, Exner M, Kramer A et al. Umsetzung der MRSA-Empfehlung der KRINKO von 1999 - Aktuelle Hinweise des Vorstands der DGKH. Hyg \& Med 2009; 34: 90 - 101

6 Chaberny IF, Wriggers A, Behnke M et al. Antibiotikaresistenz: Präventionsmaßnahmen deutscher Krankenhäuser bei MRSA: Ergebnisse einer Umfrage unter 134 Krankenhäusern im Rahmen des MRSAKISS-Moduls. Dtsch Ärztebl 2010; 107: 631 - 637

7 Geiss HK. Deutsche Kliniken vernachlässigen die Hygiene nicht. Arzt \& Krankenhaus 2010; 83: 302 305

8 Schulze-Röbbecke R. Standardmaßnahmen zur Prävention nosokomialer Infektionen - Standardhygiene, Basishygiene. Krankenhaushyg up2date 2009; 4: $193-205$

9 Kirkland KB. Taking off the gloves: Toward a less dogmatic approach to the use of contact isolation. Clin Infect Dis 2009; 48: 766 - 771 